ISSN 2693-2504

\title{
Infection Prevention in the ICU
}

\section{Efstathios Koutsostathis}

$\begin{array}{ll} & \text { *Correspondence authors } \\ & \text { Efstathios Koutsostathis } \\ \text { Kerameikos Health Center, Greece } & \text { Kerameikos Health Center } \\ \text { Greece }\end{array}$

Submitted : 14 Apr 2021 ; Published : 12 May 2021

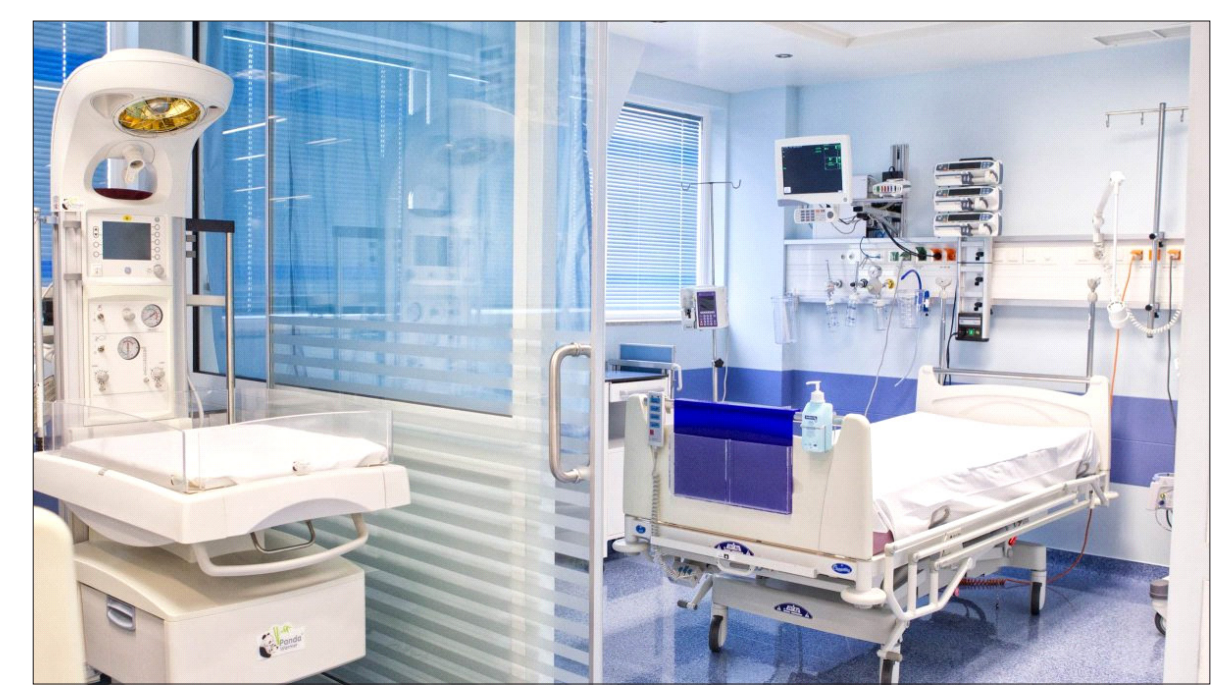

Athens

\begin{abstract}
Hospital-acquired infections are among the most significant issues within the healthcare system, both in Greece and abroad. This is because they are associated with severe morbidity and mortality. As a rule, in Intensive Care Units (ICU), hospital-acquired infections are caused by multidrug-resistant bacteria. The spread of infections by multidrug-resistant bacteria occurs in steps. Step one is usually considered to be colonisation of the ICU hostpatient via indirect contact. A transmission vehicle in these cases are the hands of healthcare professionals. The main infections in this category are the ones that affect the respiratory system, followed by bloodstream infections, mainly through endovascular catheters, and urinary tract infections. Therefore, some simple measures can limit the spread of infections, improving the clinical outcomes for hospitalised patients. These include following hand hygiene, ensuring that the medical and nursing staff change disposable gloves, keeping the ICU areas extremely clean and keeping together hospitalised patients who are colonised by the same multidrug-resistant bacteria. However, many healthcare professionals fail to consistently comply with these guidelines, which leads to the spread of multidrug-resistant bacteria, and increased morbidity and mortality.
\end{abstract}

\section{Introduction}

Around $50 \%$ of patients hospitalised in ICUs are affected by hospital-acquired infections. A hospital-acquired infection is an infection that develops 48 hours after being admitted to the ICU. The corresponding rate for patients hospitalised in wards is much lower, close to $5 \%$. It is very important to investigate the reasons hospital-acquired infections develop in the ICU at an increased frequency. These reasons relate to the host, meaning the patient, given that apart from co-morbidities, gravely ill patients have a severe clinical condition, they are at a critical point in terms of their survival and their main body functions, such as respiratory or cardiac, require mechanical support. On the other hand, during hospitalisation in the ICU, patients undergo frequent and required interventions, which bypass the defence barriers, such as the skin, when placing a central venous line, or the nasal mucosa, when placing a feeding tube (levin). In addition, administration of sedation suppresses the cough reflex, and the endotracheal tube becomes a direct access route for pathogens to reach the lower respiratory system from the oral cavity [1,2]. 
ICU infections from multidrug-resistant bacteria

These infections are split into three main categories:

- Respiratory tract infections

- Bloodstream infections

- Urinary tract infections

\section{Respiratory tract infections}

The two main hospital clinical entities linked to the pathology of the respiratory system in the ICU are ventilator-associated tracheobronchitis (VAT) and ventilator-associated pneumonia (VAP).

- VAT affects around $10 \%$ of ICU patients. It is an inflammation of the bronchial epithelium caused by infection and is diagnosed at least 48 hours after endotracheal intubation. The clinical presentation includes fever and purulent bronchial secretions, without radiological imaging findings of lung parenchyma pathology. Certain researchers consider VAT to be the intermediate stage between colonisation of the respiratory epithelium by bacteria and VAP. VAT treatment involves short-term antimicrobial regimens or inhaled antimicrobials as monotherapy. The suitable antimicrobial is selected based on the antibiogram [3].

- VAP is an infection of the lower respiratory tract, which develops two days after endotracheal intubation and subsequent mechanical ventilator support. Its diagnosis requires a combination of clinical and laboratory findings, such as temperature increase, tachypnoea or dyspnoea, presence of non-musical crackles during auscultation of the lung parenchyma, purulent bronchial secretions and accompanying leukocytosis. Radiological imaging with presence of condensed foci in the lung parenchyma may confirm initial suspicions. Isolation of the pathogen in the bronchial secretions or the bronchoalveolar lavage contributes to final diagnosis.

The bacteria that colonise the upper airways are the ones that can potentially cause VAP under certain conditions. This colonisation may possibly relate to:

- The normal flora of the patient.

- The normal flora of the patient, but modified due to hospitalisation.

- Hospital pathogens that are transmitted from patient to patient mainly via the hands of the nursing and medical staff, or even via contaminated equipment.

VAP treatment includes the administration of the so-called advanced antibiotics, which include antipseudomonal cephalosporins and quinolones, piperacillin-tazobactam, and, in the event of possible Gram-positive bacteria, linezolid or vancomycin. Treatment is individualised based on the antibiogram [4].

\section{Bloodstream Infections}

As a rule, they develop two days after being admitted to the ICU. They are separated into primary and secondary. The former, which appear more frequently, do not have a definite primary source and are usually due to the central venous catheter or other type of endovascular catheter. They may also be due to rupture of the intestinal obstruction. The secondary ones are the result of focal infection complication. Diagnosis is made through blood cultures, while treatment constitutes a medical emergency and, until the antibiogram is received, it is determined by the microbiological history of the patient and their current clinical condition, which also relates to the local microbiology of the specific infections. It generally includes advanced antimicrobials [5].

\section{Urinary Tract Infections}

The infections in this category make up $40 \%$ of all ICU infections. They have a particular epidemiological character, given that they constitute a substantial microbiological reservoir. They are separated into:

- Catheter-associated

- Asymptomatic bacteriuria

- Catheter-associated bacteriuria

Symptomatology includes fever with possible chills - in the case of pyelonephritis - fatigue, frequent urination and dysuria, as well as very frequent deterioration in the level of communication and/or lethargy.

The bacteria reach the urinary bladder through either the intraluminal or the extraluminal route of the catheter. The main bacteria that cause urinary tract infections are E. coli, but also other intestinal bacteria, such as Klebsiella spp, Enterobacter spp, Proteus mirabilis, Pseudomonas aeruginosa, Acinetobacter baumannii, etc.

In terms of treatment, asymptomatic bacteriuria does not require treatment. On the other hand, the true urinary tract infections require drastic antimicrobial treatment, given that they are very often complicated by sepsis. Antimicrobial treatment includes beta-lactams, preferably with antipseudomonal action, quinolones, aminoglycosides, colistin, fosfomycin, etc. The ICU microbiology and patient colonisation are significant for accurately determining treatment, while the antibiogram plays a determining role. In addition, it is very important to remove the urinary catheter seven or more days after insertion [6].

\section{Dispersion of Pathogenic Microorganisms}

Infections in the ICU are usually caused by bacteria endemic to the Unit. These bacteria are mainly transmitted in three ways:

- Through contact

- Through droplets

- Through airborne dispersion

In an ICU, the most significant is contact transmission, which indirectly occurs through healthcare professionals, who are a pathogen-transmission vehicle from patient to patient, but also through inanimate objects, such as diagnostic tools. It seems that a large percentage of infections, around $30 \%$, are transmitted in this manner. 
Ways to Prevent Infection Transmission in the ICU

A series of measures are used to reduce the incidence of hospital-acquired infections. They include complete prevention measures and contact-transmission prevention measures, which greatly overlap. The prevention measures are:

- Hand hygiene, which includes thorough hand washing, and use of chlorhexidine solutions and idonine antiseptics.

- Use of gloves, apron and mask by the medical and nursing staff when coming into contact with patients.

- Hospitalisation area cleaning protocol.

- Isolated hospitalisation and respiratory hygiene protocol for patients with infectious diseases, either respiratory or in general.

- Safe cardiopulmonary resuscitation and safe sharps handling protocol.

More specifically

- Hand hygiene: As early as the 19th century, Semmelweis' observations as to hand disinfection in obstetrical clinics created controversy with the medical status quo at the time. Even these days, though, and despite the fact that Semmelweis' findings have been fully confirmed, the medical and nursing staff does not seem to be fully complying with the hand hygiene rules within hospital settings. The acceptable hand hygiene measures include thorough hand washing with soap and water for quite an extended period of time, at least 5 minutes, so the areas between the fingers may be disinfected. In addition, cleaning with alcohol-based sanitizers is considered necessary. Hand washing is imperative every time a doctor or nurse is about to or has just come into contact with an ICU patient.

- Isolated hospitalisation: It is further distinguished into protective isolation and source isolation. In the first case, the patient is isolated to be protected against the possible spread of bacteria. This is performed mainly due to immune system deficiencies, such as neutropenia or splenectomy. In the second case, the rest of the patients are protected against the potential spread of a bacterial agent from a patient affected by it, as, for example, in the case of active tuberculosis, HIV infection or viral meningitis. When separate hospitalisation is not possible, patients with similar microbiological characteristics are hospitalised in the same ward, e.g. patients suffering from H1N1 flu. Other cases that require isolated hospitalisation include diseases associated with airborne transmission via droplets, such as meningococcal meningitis and streptococcal pharyngitis. In these cases, transmission can occur via tiny droplets during simple speech. For this reason, it is best to keep a distance of at least 2-3 metres from the patient. The use of a mask is also important, both for the patient and for the nursing staff when performing their duties in the room of a patient suffering from an infectious disease.

The key trait of the ICU is the personal care each patient receives, in a specially designed area, which, apart from the bed and ventilator, includes a special cabinet with the necessary hospitalisation materials, gloves, sterile gauze, injections, and drip and pharmaceutical treatment preparation area, so as to reduce the risk of bacteria transmission from patient to patient. In addition, three patients at most correspond to each ICU nurse.

- Use of chlorhexidine: The use of chlorhexidine solution has been proven to significantly reduce the incidence of transmission of pathogens and hospital-acquired infections, according to a number of relevant studies [7].

\section{Prevention of the most common ICU Infections}

\section{- Ventilator-associated pneumonia and ventilator-asso- ciated tracheobronchitis}

The recording of the local epidemiology of both VAP and VAT plays a major role in infection prevention. This can be achieved with the use of protocols, based on which the incidence of respiratory infections from various pathogens - these are generally known as multidrug-resistant bacteria in ICUs could be updated weekly.

On the other hand, educating the medical and nursing staff on prevention methods and application of simple daily practices could help. Such practices are:

- $\quad$ Placing patients in the semi-recumbent position, at around a 30-45' angle, especially during feeding, to avoid the possibility of aspiration pneumonia.

- Practising proper oral hygiene, which decreases the bacterial load, limiting the possibility of bacteria entering the lower respiratory system.

- Opting for non-invasive mechanical ventilation, especially in situations where it is indicated, such as pulmonary oedema and exacerbation of chronic obstructive pulmonary disease. This limits the spread of bacteria in the oral cavity and the upper respiratory tract at the lower part of the endotracheal tube. In addition, when invasive ventilation is necessary, especially in cases that will remain ventilated for a long time, it is best to choose an endotracheal tube with subglottic suctioning lumen.

- Monitoring the pressure of the endotracheal tube air chamber. The pressure must be $20-30 \mathrm{mmHg}$, so as to ensure proper sealing and avoid the risk of ischemic tracheal mucosal injury [8].

Prevention of bloodstream and urinary tract infections in the ICU

The significance of applying simple practices for bloodstream infection prevention in ICUs was becoming evident as early as the beginning of the previous decade. These practices are:

- Applying aseptic conditions (surgical conditions) when placing central venous catheters, with the use of chlorhexidine.

- Removing all unnecessary catheters on time.

- Avoiding the femoral vein for catheterisation.

- Implementing hand hygiene daily when reaching the entrance, with the use of chlorhexidine [9]. 
In terms of urinary tract infection prevention, it is deemed necessary to limit catheterisations to the absolutely necessary ones, follow sanitation rules every time a urinary catheter is placed and remove the urinary catheter as soon as it is not absolutely necessary. Other prevention measures include avoiding backflow of urine into the urinary bladder, and keeping the urine management system aseptic and sealed [10].

\section{Discussion/Conclusions}

The prevention of hospital-acquired infections, especially in an ICU setting, are a top priority for the healthcare system and staff. This arises from the fact that a significant number of hospitalisations is complicated by hospital-acquired infections, resulting in increased hospitalisation periods at best or even death due to septic shock combined with complications from co-morbidities. This applies more so in the ICU because endemic pathogens are generally multidrug-resistant, so possible infection by them often proves deadly or, in all events, extremely burdensome as to the inpatients' disease progress.

The fact that prevention of infection transmission is possible through daily practices that can be followed easily by all the staff causes significant distortion in the area of healthcare: In many cases, an oversimplified perception of the almost magical abilities of doctors and pharmaceutical preparations seems to prevail, while it has not yet been understood that the treatment, even of the gravely ill patients, does not so much depend on the application of complex and sophisticated therapies (although this is important too) as on following guidelines and practices that foster proper hospitalisation and hygiene. Everyday experience in hospital settings shows that cleaning the hospitalisation areas, even with domestic antiseptic solutions, is just as important as following the medical instructions to the letter. However, the fact is that the situation has improved significantly compared to the past. Nowadays, immense significance is given to applying the hygiene rules in the hospital areas and especially in the ICUs.

So from the moment that rules exist and they are the object of study by medical and nursing staff, it is up to them to follow them as best possible. This is expected to improve the progress of patient hospitalisation, lead to fewer hospitalisations, reduce the tolerance levels of bacteria, even with non-advanced antimicrobials, and significantly reduce mortality. Whatever the case may be, the significance of personal responsibility for the best possible result is of major significance for the recovery and survival of the patient, as well as for their quality of life after hospitalisation.

\section{References}

1. Klevens RM, Edwards JR, Richards CL, et al, (2007) Estimating Health Care Associated Infections and deaths in US Hospitals ,2002,Public Health Rep.2007;122: 160166.

2. Apostolopoulou E, Raftopoulou V, Filntisis G, et al, (2013) Surveilance of Device Associated Infection Rates and Mortality in 3 Greek Intensive Care Units. Am J Crit Care 2013;22; e12-e20.

3. Craven DE, Hjalmarson KI (2010) Ventilatot assosiated tracheobronchitis and pneumonia:thinking outside the box. Clin.Inf. Dis. 2010;51 Suppl 1: S59-66.

4. Liapikou A, Rosales-Mayor E, Torres A (2014) The management of severe community acquired pneumonia in the intensive care unit. Expert Rev.Resp.Med.2014;8: 293303.

5. Mermel LA, Allon M, Bouza E, et al, (2009) Clinical practice guidelines for the diagnosis and management of intravascular catheter-related infection:2009 Update by the Infectious Diseases Society of America. Clin.Infect. Dis. 2009;49: 1-45.

6. Hooton TM, Bradley SF, Cardenas DD, et al (2010) Diagnosis, prevention and treatment of catheter-associated urinary tract infections in adults. International clinical practice guidelines from the Infectious Diseases Society of America. Clin. Infect. Dis. 2010;50: 625-663.

7. Tscudin-sutter S, Pargger H, Windmer AF (2010) Hand hygiene in the intensive care unit. Crit. Care Med.2010.

8. Lim WS, Baudouin S, George RC, et al (2009) BTS guidelines for the management of community acquired pneumonia in adults:2009. Thorax 2009;64: 31-55.

9. Pronovost P, Needham D, Berenholtz S, et al, (2006) An intervention to decrease catheter-related bloodstream infections in the ICU.N. Engl.J.Med.2006;355: 2725-2732.

10. Chant C, Smith OM, Marshall JC, Friedrich JO (2011) Relationship of catheter-associated urinary tract infection to mortality and length of stay in critically ill patients:a systemic review and meta-analysis of observational studies. Crit.Care Med.2011;39: 1167-1173.

Copyright: (C2021 Efstathios Koutsostathis. This is an open-access article distributed under the terms of the Creative Commons Attribution License, which permits unrestricted use, distribution, and reproduction in anymedium, provided the original author and source are credited. 